\title{
Control of Isocitrate Lyase in Nocardia salmonicolor (NCIB9701)
}

\author{
By F. SIMA SARIASLANI,* A. W. WESTWOOD AND I. J. HIGGINS \\ Biological Laboratory, University of Kent, Canterbury $\mathrm{CT}_{2}{ }_{7} \mathrm{NJ}$, Kent
}

(Received 4 April 1975; revised 7 June 1975)

\begin{abstract}
SUMMARY
Nocardia salmonicolor, grown on acetate, commercial D,L-lactate or hydrocarbon substrates, has high isocitrate lyase activities compared with those resulting from growth on other carbon sources. This presumably reflects the anaplerotic role of the glyoxylate cycle during growth on the former substrates. Amongst a variety of compounds tested, including glucose, pyruvate and tricarboxylic acid cycle intermediates, only succinate and fumarate prevented an increase in enzyme activity in the presence of acetate. When acetate (equimolar to the initial sugar concentration) was added to cultures growing on glucose, there followed de novo synthesis of isocitrate lyase and isocitrate dehydrogenase, with increases in growth rate and glucose utilization, and both acetate and glucose were metabolized simultaneously. A minute amount of acetate $(40 \mu \mathrm{M})$ caused isocitrate lyase synthesis (a three-fold increase in activity within $3 \mathrm{~min}$ of addition) when added to glucose-limited continuous cultures, but even large amounts added to nitrogen-limited batch cultures were ineffective. Malonate, at a concentration that was not totally growth-inhibitory (I $\mathrm{mm}$ ) prevented the inhibition of acetate-stimulated isocitrate lyase synthesis by succinate, but fumarate still inhibited in the presence of malonate. Phosphoenolpyruvate is a noncompetitive inhibitor of the enzyme (apparent $K_{i} \mathrm{I} \cdot 7 \mathrm{~mm}$ ).

The results are consistent with the induction of isocitrate lyase synthesis by acetate or a closely related metabolite, and catabolite repression by a C-4 acid of the tricarboxylic acid cycle, possibly fumarate.
\end{abstract}

\section{INTRODUCTION}

For Escherichia coli, there is strong evidence that both the synthesis and activity of threo$D_{s}$-isocitrate lyase are controlled by the cytoplasmic concentration of phosphoenolpyruvate (PEP) or a closely related metabolite. PEP is a non-competitive inhibitor of the enzyme and may itself be the repressor metabolite (Kornberg, I966). It is not surprising that PEP should have this role since it may be regarded as a major end-product of the glyoxylate cycle and the starting material for the synthesis of many compounds. Under conditions where the anaplerotic role of the glyoxylate cycle is unnecessary in this organism, such as growth on C-3, C-4 or C-6 compounds, high levels of PEP in the cytoplasm might be expected.

Evidence for this control mechanism in other microbes, particularly Achromobacter sp., Acinetobacter sp. and Neurospora crassa is more equivocal. Synthesis of isocitrate lyase was not inhibited by the presence of succinate in cultures of Achromobacter d-I 5 growing on acetate (Rosenberger, 1962). Also, the presence of acetate in cultures growing on succinate caused enzyme synthesis. These results were quite different from those obtained with $E$. coli and Rosenberger interpreted them as an apparent inductive effect of acetate. However, isocitrate lyase synthesis is rapid during growth of this micro-organism on C-3 compounds (Kornberg, Dennis \& Wilson, 1964). In addition, Kornberg et al. (1964) found that the

* Present address: Biology Department, Faculty of Science, National University of Iran, Tehran, Iran. 
inducer-like effect was lessened or abolished by reducing the rates of removal from central metabolic pathways of intermediates required for the biosynthesis of component buildingblocks of macromolecules. Achromobacter d-I5 grows on tricarboxylic acid cycle intermediates and their direct metabolic precursors but not on glucose, glycerol or other substances catabolized via PEP. In view of this and the absence of pyruvate kinase in cell-free preparations, Kornberg et al. (I964) suggested that high isocitrate lyase activities during growth on C-3 compounds may be due to an inability to form PEP from pyruvate.

The isocitrate lyase activity found in an Acinetobacter sp. during growth on an equimolar mixture of acetate and succinate represented $75 \%$ of that after growth on acetate alone (Bell \& Herman, 1967). Also, addition of acetate to cultures growing on either succinate or L-malate caused rapid synthesis of isocitrate lyase. Growth on pyruvate alone resulted in a significant increase in activity even though this bacterium contains PEP synthase levels comparable to those found in E. coli (Herman \& Bell, I970). The authors concluded that, in this Acinetobacter strain, C-4 intermediates of the tricarboxylic acid cycle may be 'coarse' control metabolites, regulating the rate of operation of the glyoxylate cycle. Similarly, Beever (1975) concluded that in Neurospora crassa, a C-4 intermediate common to the tricarboxylic and glyoxylate cycles represses isocitrate lyase synthesis. The possibility of acetate induction was discounted since growth on glucose-acetate mixtures did not lead to high levels of the enzyme. Earlier work with this micro-organism had suggested regulation by a glycolytic intermediate or derivative (Flavell \& Woodward, I97I). There is also some evidence that in microbes other than Enterobacteria, PEP may not be the most important metabolite involved in 'fine' control of isocitrate lyase. For example, in Candida guilliermondii other intermediates such as oxaloacetate are more powerful inhibitors (Hildebrandt \& Weide, 1974).

During studies of the metabolism of long-chain I-phenylalkanes by Nocardia salmonicolor, the organism was found to contain high isocitrate lyase activity even when the alkane sidechain and the aromatic ring were utilized simultaneously (Sariaslani, Harper \& Higgins, 1974). Under these circumstances C-3 and C-4 intermediates, in addition to acetate, are fed into the central metabolic pathways. Isocitrate lyase activity on rich media, however, was very low. It was these observations, indicating that regulation of isocitrate lyase synthesis in this bacterium may be different from that in other micro-organisms so far studied, that prompted the present investigation. A preliminary account of part of this work has been published (Higgins \& Sariaslani, 1973).

\section{METHODS}

Micro-organism, source, maintenance and culture. Nocardia salmonicolor (NCIB970 I) was obtained from the National Collection of Industrial Bacteria, Aberdeen. It was maintained on nutrient agar slopes (Oxoid; $2.8 \%, \mathrm{w} / \mathrm{v}$ ) and grown at $30{ }^{\circ} \mathrm{C}$ in mineral salts medium (Davis \& Raymond, I96I) containing a trace metal mixture (I ml/1; Bauchop \& Elsden, I960), filter-sterilized carbon sources being added aseptically. Inocula were grown on $500 \mathrm{ml}$ nutrient broth (Oxoid No. $2 ; 2.5 \%, \mathrm{w} / \mathrm{v}$ ) in conical flasks (2 l) plugged with cotton wool, incubated on a gyrotory shaker at $30^{\circ} \mathrm{C}$, harvested in exponential phase, washed twice in sterile, sodium-potassium phosphate buffer ( $10 \mathrm{mM}, \mathrm{pH} 7^{\circ}$ ) ) and resuspended in the same buffer. For growth in batch culture on a single substrate, the carbon source was supplied at $0.5 \%(\mathrm{w} / \mathrm{v})$; in mixed substrate experiments the first carbon source mentioned in the text was supplied, at $0.5 \%(\mathrm{w} / \mathrm{v})$, the second being equimolar to the first.

Batch cultures ( $1 \cdot 0$ to 2.5 l) were grown with an air flow of I culture volume $/ \mathrm{min}$, either in an impeller-agitated fermenter $(3.01$ capacity; L.H. Engineering, Stoke Poges, Buckinghamshire), the stirring rate being 500 to $700 \mathrm{rev} . / \mathrm{min}$, or in magnetically stirred culture 
vessels (L.H. Engineering or Quickfit, Jobling Ltd, Stone, Staffordshire; I 1 capacity). Continuous cultures, in a magnetically stirred vessel (Quickfit; $1 \cdot 21$ culture volume, air flow rate $100 \mathrm{ml} / \mathrm{min}$ ), were glucose-limited at a dilution rate of 0.023 to $0.025 \mathrm{~h}^{-1}$. The concentration of glucose in the dilution medium was $0.4 \%(\mathrm{w} / \mathrm{v})$.

Growth measurement. Turbidity in an EEL colorimeter was related to dry weight by using a standard curve prepared with glucose-grown organisms.

Preparation of cell-free extracts. Organisms were harvested by centrifugation $(23000 \mathrm{~g}$, $\mathrm{I} \mathrm{h}, 4{ }^{\circ} \mathrm{C}$ ), washed twice with sodium-potassium phosphate buffer (10 mM, $\mathrm{pH} 7 \cdot 0$ ), and resuspended in the same buffer ( 2 to $5 \mathrm{ml}$ ) before sonicating for $2 \mathrm{~min}$ in an MSE sonicator (type $150 \mathrm{~W}$ ) whilst cooling in ice. The resulting suspensions were centrifuged at $38000 \mathrm{~g}$ for 15 min at $4{ }^{\circ} \mathrm{C}$ and the pellet discarded.

Protein assay. The protein concentration in extracts was determined by the method of Lowry et al. (I95I) with bovine serum albumin (fraction V; Sigma) as a standard.

Enzyme assays. Isocitrate lyase (EC. 4.I.3.I) was measured as described by Dixon \& Kornberg (1959), isocitrate dehydrogenase (EC. I . I . I .4I) by the method of Horecker \& Kornberg (1948), and phosphoenolpyruvate synthase by the method of Cooper \& Kornberg (1967).

Chemical estimations. The glucose concentration in culture supernatants was determined by the method of Hultmann (1959) using Boehringer biochemical test combinations (Boehringer Corp. Ltd, London). Acetate was measured in acidified culture supernates $(\mathrm{pH} 2 \cdot 0)$ by gas chromatography, using a coiled-glass column ( $1.83 \mathrm{~m} \times 0.4 \mathrm{~cm}$ internal diameter) containing Porapak Q (80 to I00 mesh; Pye Unicam Ltd, Cambridge). A Pye model 104 gas chromatograph fitted with a flame ionization detector was used. Conditions were as follows: carrier gas flow rate, $70 \mathrm{ml} / \mathrm{min}$; oven temperature $180^{\circ} \mathrm{C}$; detector temperature, $230{ }^{\circ} \mathrm{C}$.

Samples of commercial lactate were analysed for acetate content using a similar gas chromatographic system, except that the column was packed with $10 \%(\mathrm{w} / \mathrm{v})$ polyethylene glycol $20 \mathrm{M}$ on 100 to I 20 mesh diatomite A-AW (Pye Unicam Ltd). Conditions were: carrier gas flow rate, $60 \mathrm{ml} / \mathrm{min}$; oven temperature $125^{\circ} \mathrm{C}$; detector temperature, $200{ }^{\circ} \mathrm{C}$.

Chemicals. ATP, NADH, sodium acetate, sodium fumarate, sodium D,L-isocitrate, sodium D,L-lactate, sodium L-malate, oxaloacetic acid and sodium pyruvate were obtained from Sigma, D,L-alanine, D,L-glutamate and glucose from BDH, and trisodium citrate and sodium succinate from Fisons Scientific Apparatus, Loughborough, Leicestershire. All other chemicals were of the highest commercial quality.

\section{RESULTS AND DISCUSSION}

\section{Effect of carbon source on growth rate and isocitrate lyase levels}

The mean generation times during batch-culture exponential growth of $N$. salmonicolor on a variety of carbon sources are shown in Table $\mathrm{I}$. The organism grew most rapidly on acetate, nutrient broth and the hydrocarbon substrates. Growth on acetate together with a second carbon source was significantly slower than that on acetate alone, except when the second substrate was glucose.

The maximum specific activities of isocitrate lyase during adaptation to these various substrates are also shown in Table I. Growth on acetate (Fig. I $a$ ), D,L-lactate or the hydrocarbon substrates resulted in high enzyme activities compared with those attained during growth on any of the other single substrates. High activities in micro-organisms growing on $n$-alkanes have been reported previously (Trust \& Millis, 1970) and this reflects the catabolism of these compounds via acetyl-CoA. Activities during growth on I-phenylalkanes were 
Table I. The effect of growth substrate on the activity of isocitrate lyase in extracts of $N$. salmonicolor

Isocitrate lyase activity was measured in cell-free extracts prepared from organisms harvested at various stages of the growth cycle and the maximum values recorded are tabulated.

\begin{tabular}{|c|c|c|}
\hline Substrate & $\begin{array}{c}\text { Maximum specific activity } \\
\text { of isocitrate lyase } \\
\text { (nmol/mg protein } / \mathrm{min} \text { ) }\end{array}$ & $\begin{array}{c}\text { Mean generation } \\
\text { time (h) }\end{array}$ \\
\hline Acetate & 296 & 5 \\
\hline Pyruvate & 5 & 16 \\
\hline Glucose & 10 & 12 \\
\hline D,L-Lactate & 224 & 10 \\
\hline D,L-Alanine & 4 & 12 \\
\hline D,L-Glutamate & 3 & 20 \\
\hline Nutrient broth & Io & 5 \\
\hline \multicolumn{3}{|c|}{ Tricarboxylic acid cycle intermediates } \\
\hline Citrate & 2 & 12 \\
\hline Fumarate & I I & 8 \\
\hline D,L-Isocitrate & 2 & 22 \\
\hline L-Malate & 8 & 8 \\
\hline Oxaloacetate & 2 & 10 \\
\hline Succinate & IO & 8 \\
\hline \multicolumn{3}{|c|}{ Acetate together with an equimolar concentration of: } \\
\hline Citrate & 47 & I I \\
\hline Fumarate & 10 & 8 \\
\hline Glucose & 310 & 5 \\
\hline D,L-Glutamate & 32 & 20 \\
\hline D,L-Isocitrate & 16 & 22 \\
\hline L-Malate & 112 & 8 \\
\hline Oxaloacetate & 294 & Io \\
\hline Pyruvate & 265 & 7 \\
\hline Succinate & IO & 8 \\
\hline \multicolumn{3}{|l|}{ Hydrocarbons } \\
\hline Hexadecane & 160 & 5 \\
\hline I-Phenyldodecane & IIO & 6 \\
\hline I-Phenylnonane & 55 & 6 \\
\hline
\end{tabular}

somewhat lower than during growth on acetate, but greater than in glucose- or nutrient broth-grown organisms (II- and 5.5-fold increases for I-phenyldodecane- and I-phenylnonane-grown organisms, respectively).

The high enzyme activity during growth on D,L-lactate appears anomalous, especially since activity is very low during growth on the closely related substrates pyruvate and alanine. The result may be due to contamination of commercial lactate with acetate, because very little acetate is required to cause enzyme synthesis (Fig. 6). Indeed, gas chromatographic analysis of $\mathrm{D}, \mathrm{L}-, \mathrm{L}(+)$ - and $\mathrm{D}(-)$-lactate from various suppliers revealed significant amounts of acetate impurity $(0.05$ to $0.2 \%, \mathrm{w} / \mathrm{w})$. An alternative explanation is that this bacterium does not oxidize lactate via pyruvate. Lactate dehydrogenase has not been found in lactategrown organisms (Westwood and Higgins, unpublished observations) and it is possible that lactate is converted directly to acetate by an oxygenase (Sutton, 1954).

When the organism was grown on mixtures of acetate with pyruvate, glucose or tricarboxylic acid cycle intermediates, enzyme levels were usually high and similar to those seen when acetate was the sole substrate. In contrast, when the second substrate was succinate or fumarate enzyme activity remained at the normal background level. Activities were relatively low with citrate, glutamate and isocitrate, but this was associated with poor and slow 

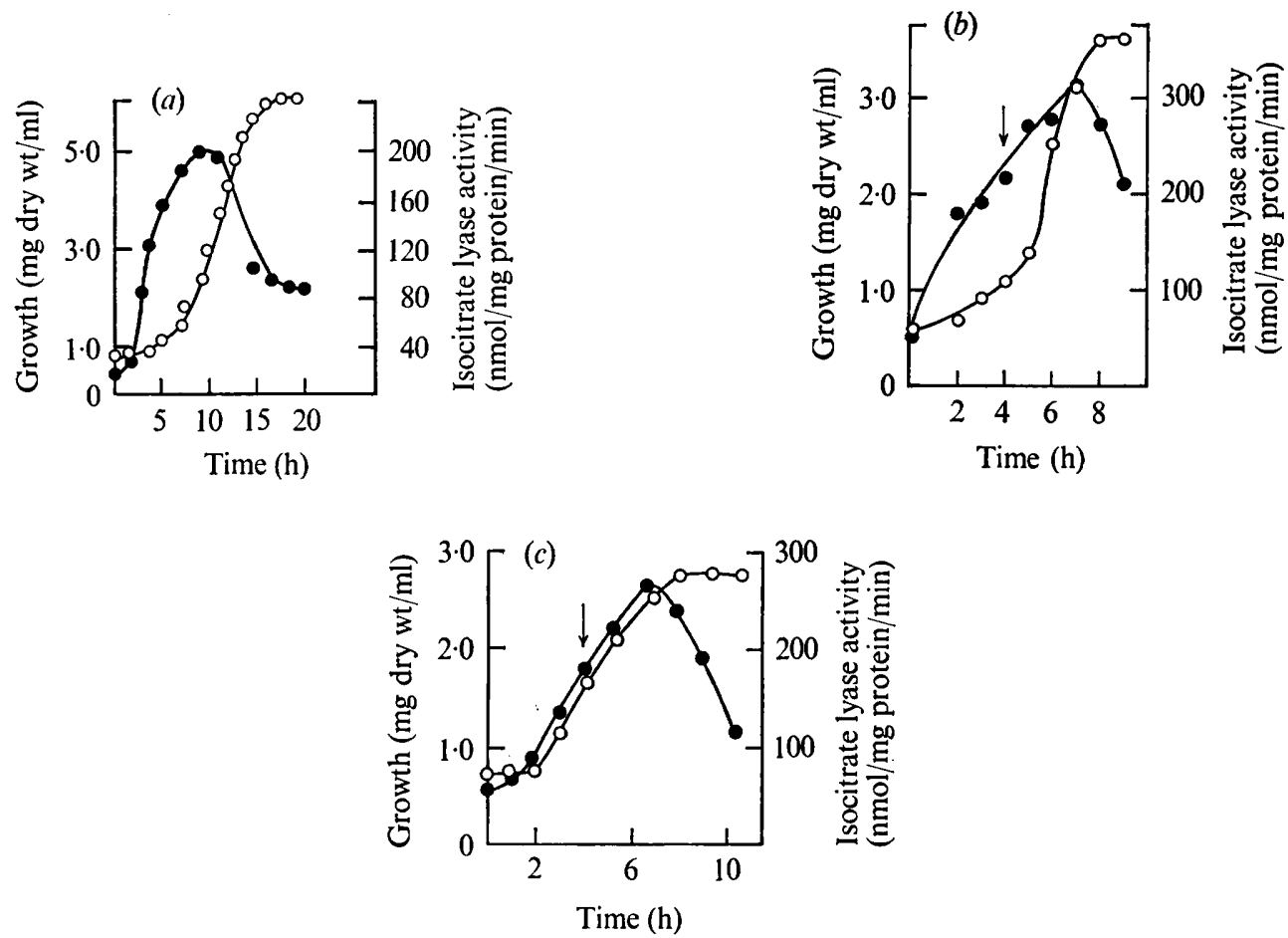

Fig. I. The effect of the addition of glucose or pyruvate on the pattern of cell-free isocitrate lyase activity in $N$. Salmonicolor growing initially on acetate. (a) No addition; $(b)$ equimolar glucose or (c) pyruvate added at time indicated by arrow. $\mathrm{O}$, Growth;, , isocitrate lyase.

growth on these compounds even in the presence of acetate (Table I). Nevertheless, the presence of acetate did cause considerable increases in enzyme activity (23-, I I- and 8-fold for citrate, glutamate and isocitrate, respectively). These results were quite distinct from those for fumarate and succinate, where acetate had no effect whatsoever.

Clearly, these results are different from those obtained with other micro-organisms. One interpretation of them would be that acetate, or a closely related metabolite such as acetyl$\mathrm{CoA}$, acts as an inducer, whilst succinate and/or fumarate act as catabolite repressor(s). However, there are other possibilities, such as specific inhibition by acetate of the transport of other potential substrates into the organism. Clarification of this phenomenon would have been facilitated by the appropriate metabolic mutants, but since these were not available it was necessary to adopt an indirect approach.

The effect on the pattern of isocitrate lyase activity of adding $C-3, C-4$ or $C-6$ compounds to batch cultures growing initially on acetate

The pattern of isocitrate lyase activity during a typical batch culture with acetate as the sole carbon source is shown in Fig. I (a). The specific activity increased rapidly during the lag phase, and reached a maximum early in the exponential phase before declining sharply towards the end of growth. Exactly the same pattern was seen with the mixed substrates (Table I) except when the second substrate was fumarate or succinate. Additions of glucose (Fig. $\mathrm{I} b$ ) or pyruvate (Fig. I $c$ ), at concentrations equimolar to the initial acetate concentration, to early exponential-phase cultures growing on acetate, were without significant effect 


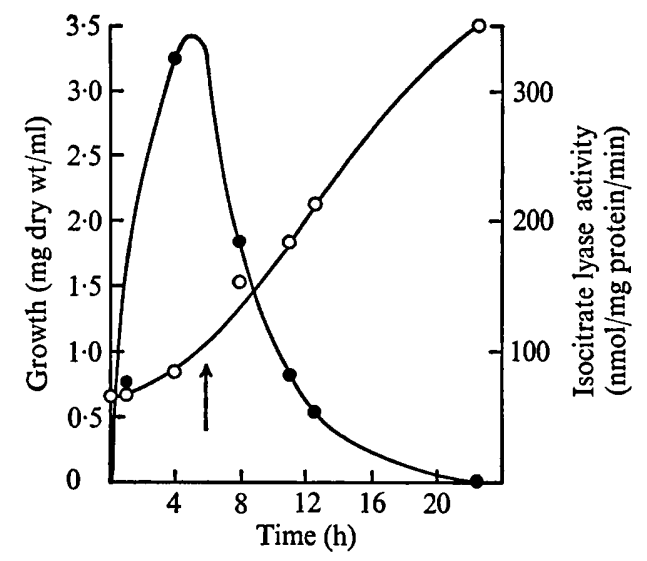

Fig. 2. Effect on isocitrate lyase activity during growth of adding equimolar fumarate (arrow) to a culture of $N$. salmonicolor growing initially on acetate. $O$, Growth; $\bullet$, isocitrate lyase.

on the pattern of enzyme activity except perhaps to delay the onset of reduction of enzyme activity towards the end of growth. If PEP or a closely related metabolite was the corepressor for isocitrate lyase synthesis such additions might be expected to cause a reduction of enzyme synthesis, especially in the case of pyruvate, as PEP-synthase activity of 100 to I $20 \mathrm{nmol} / \mathrm{mg}$ protein $/ \mathrm{min}$ was detected routinely in acetate-grown organisms. Additions of citrate, isocitrate, malate or oxaloacetate to cultures growing on acetate were similarly without effect on the pattern of enzyme synthesis. There remained the possibility that organisms growing on acetate were impermeable to these other potential carbon sources, but this was unlikely when succinate or fumarate was added, as they both caused a rapid decrease in enzyme activity after their addition. The results of fumarate addition are shown in Fig. 2.

\section{The effect on isocitrate lyase activity of adding acetate to cultures growing initially} on $C-3, C-4$ or $C-6$ compounds

The result of adding an equimolar amount of sodium acetate to a culture growing initially on pyruvate is shown in Fig. 3(a). The addition caused a rapid and substantial increase in activity within an extremely short time. Similar results were obtained on adding acetate to cultures growing on citrate, isocitrate, malate, oxaloacetate or glucose. The increases in enzyme activity varied somewhat, depending on the levels before acetate addition and on the growth rate, but were always between 20 - and 70 -fold. Addition of acetate to cultures growing on succinate (Fig. $3 b$ ) or fumarate did not result in detectable increases in the background level of the enzyme.

The effect on growth rate and substrate utilization of acetate addition to a culture growing initially with glucose as carbon source

Fig. 4 shows the results of a detailed analysis of the effects of adding an equimolar amount of sodium acetate to a culture of $N$. salmonicolor growing initially on glucose. The addition was followed by rapid increases in growth rate (halving of mean generation time from approximately 10 to $5 \mathrm{~h}$ ), isocitrate lyase activity, isocitrate dehydrogenase activity and glucose utilization. Both carbon sources were utilized simultaneously after the addition, a highly unusual finding. The patterns of activities of both enzymes that act on isocitrate were closely similar; this might reflect regulatory linkage, or perhaps the increase in isocitrate dehydro- 

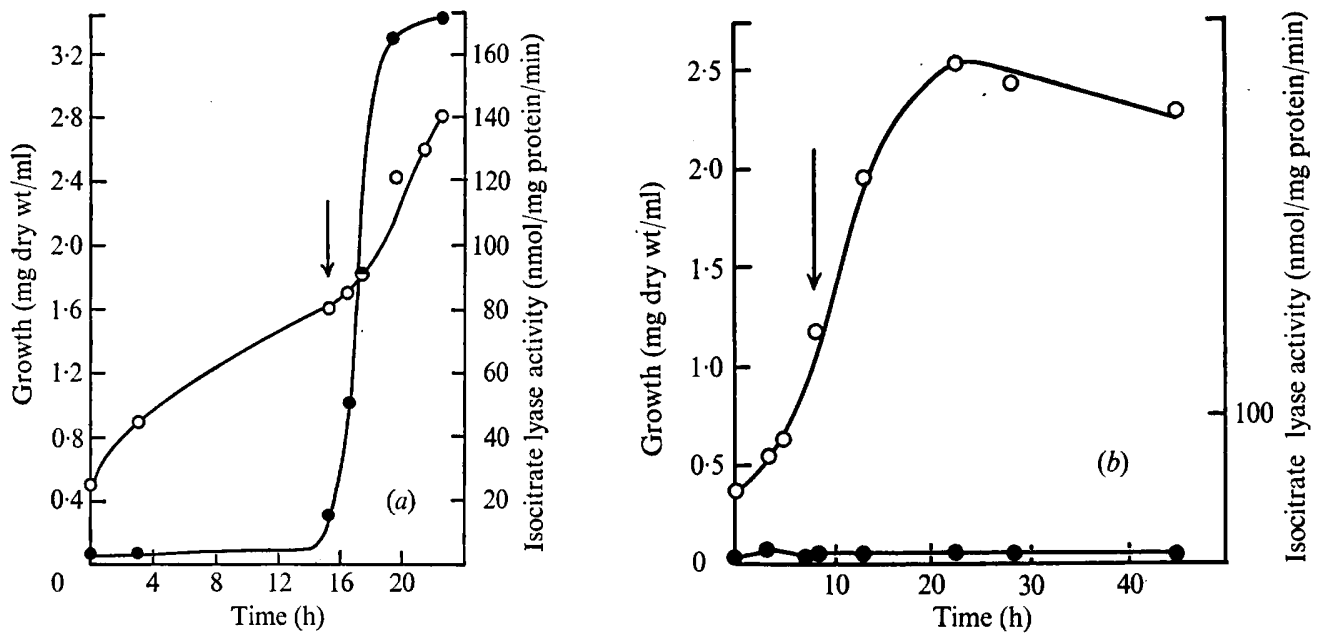

Fig. 3. Effect on isocitrate lyase activity during growth of adding equimolar acetate (arrow) to a culture of $N$. salmonicolor growing initially on $(a)$ pyruvate or $(b)$ succinate. $\bigcirc$, Growth; $\bullet$, isocitrate lyase.

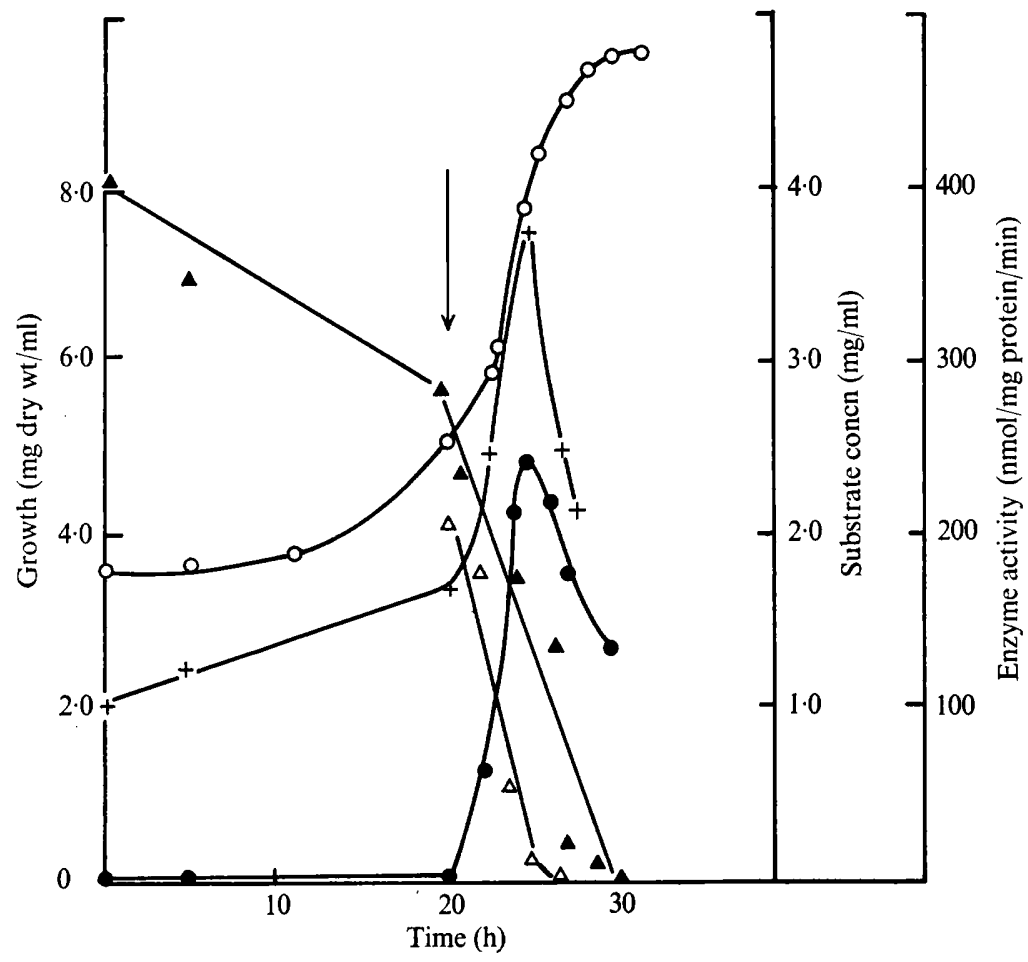

Fig. 4. The effect on enzyme levels, growth rate and utilization of substrates, of adding equimolar acetate (arrow) to a culture of $N$. salmonicolor growing initially on glucose. $\bigcirc$, Growth; $\bullet$, isocitrate lyase; + , isocitrate dehydrogenase; $\triangle$, acetate utilization; $\Delta$, glucose utilization. 


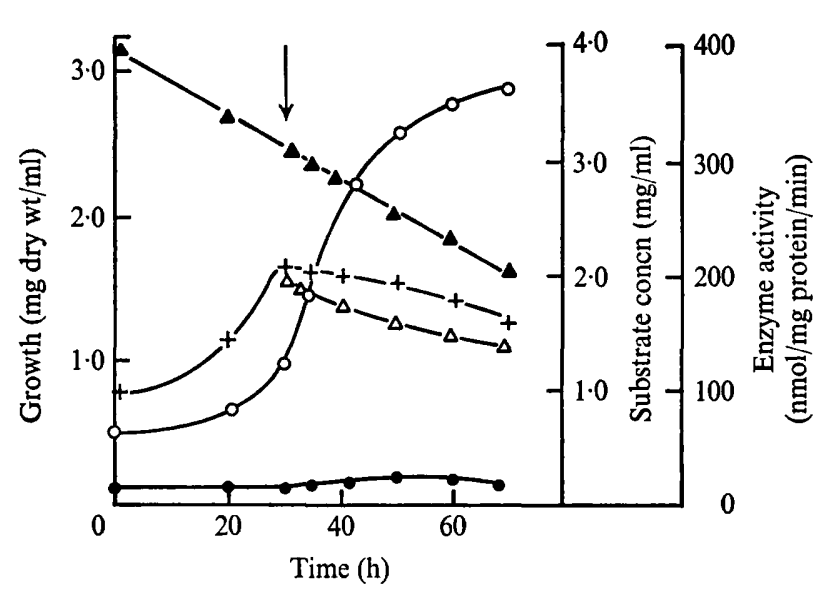

Fig. 5

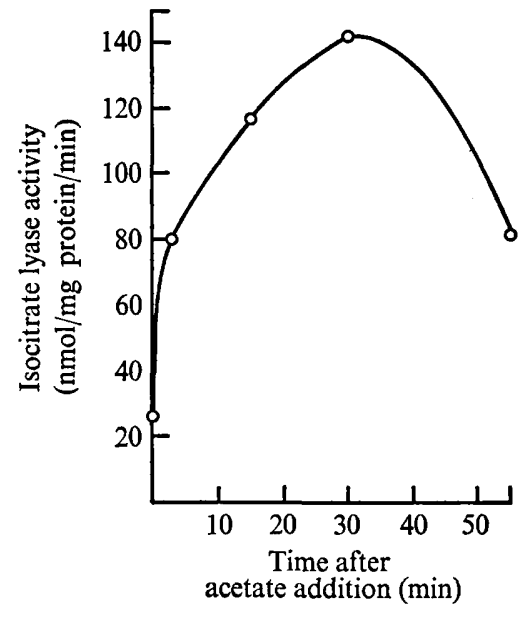

Fig. 6

Fig. 5. The effect on enzyme levels, growth rate and utilization of substrates of adding equimolar acetate plus chloramphenicol (arrow) to a culture of $N$. salmonicolor growing initially on glucose. $O$, Growth;, , isocitrate lyase; + , isocitrate dehydrogenase; $\triangle$, acetate utilization; $\boldsymbol{\Delta}$, glucose utilization. The chloramphenicol concentration in the culture was $200 \mu \mathrm{g} / \mathrm{ml}$.

Fig. 6. The effect on isocitrate lyase activity of adding a small amount of acetate to $N$. salmonicolor growing on glucose in continuous culture under conditions of substrate limitation. Acetate $(40 \mu \mathrm{M}$ final concentration) was added to a steady state culture growing on glucose (Methods). The dilution rate was $0.025 \mathrm{~h}^{-1}$.

genase activity is a function of the growth rate. The possibility that the increase in enzyme activities was due to activation of pre-existing proteins was excluded by repeating the experiment but adding chloramphenicol $(200 \mu \mathrm{g} / \mathrm{ml})$ along with the acetate (Fig. 5). Neither enzyme activity was stimulated, and as a result acetate utilization was slow; there was no stimulation of glucose utilization.

Increased biosynthesis of isocitrate dehydrogenase, resulting from the addition of acetate to a culture growing on glucose, contrasts sharply with results obtained for some other bacteria, in which growth on acetate as sole carbon source causes a reversible inactivation of a large proportion of the isocitrate dehydrogenase, which is observed as an apparent repression of enzyme activity (Bennett \& Holms, 1975). This no doubt reflects the capacity of the Nocardia to grow more rapidly on acetate than on glucose.

\section{Regulation of isocitrate lyase biosynthesis}

The possibility that acetate or a closely related compound acts as an inducer was further investigated by using a continuous culture of the micro-organism growing on glucose under conditions of substrate limitation, as described in Methods. The residual glucose concentration was $4 \mathrm{mM}$, and the results of adding acetate (as a discrete volume at zero time) to the culture at $\mathrm{I} \%$ of this glucose concentration (i.e. at $40 \mu \mathrm{M}$ ) are shown in Fig. 6 . There was an extremely rapid increase in isocitrate lyase activity (threefold within $3 \mathrm{~min}$, sevenfold within $30 \mathrm{~min}$ ). Such a dramatic effect resulting from this low concentration of acetate is most readily explained by an induction mechanism, but there are other possibilities; for example, acetate could interact with a key enzyme of intermediary metabolism causing a change in the pool size of a co-repressor, although this seems less likely in view of the short response time. 
In the absence of mutants, further evidence could only be obtained by a detailed analysis of the immediate effect (if any) of adding trace amounts of acetate on the pool sizes of the components of central metabolic pathways.

The possible role of fumarate and/or succinate as catabolite repressor(s) of isocitrate lyase synthesis was investigated further by two types of experiment. In the first, the micro-organism was grown on glucose under conditions of nitrogen limitation (nitrogen source, urea at $0.0 \mathrm{I} \%, \mathrm{w} / \mathrm{v})$. That nitrogen limitation had been established was indicated by a large decrease in the protein:dry weight ratio, although there was little effect on growth rate. When equimolar acetate was added to such cultures during the growth phase, the usual increase in isocitrate lyase activity did not occur. This may be interpreted as being due to accumulation of a repressor metabolite resulting from nitrogen limitation restricting the overall rate of biosynthesis. The second type of experiment involved malonate addition. Malonate (I mM) was added to an exponential-phase culture growing on succinate. (The organism does not grow on malonate.) This reduced the growth rate to $10 \%$ of that on succinate alone. When acetate (equimolar to the initial succinate concentration) was then added, isocitrate lyase activity increased rapidly (about tenfold). Malonate therefore reversed the inhibitory effect of succinate on enzyme synthesis in response to acetate. Since malonate is known to inhibit succinic dehydrogenase, this experiment suggests that fumarate, rather than succinate, may be the catabolite repressor. This hypothesis is further supported by analogous experiments in which malonate (I $\mathrm{mm}$ ) was added to cultures growing on fumarate. The growth rate was similarly reduced, but in contrast there was no isocitrate lyase synthesis in response to acetate addition.

The regulation of isocitrate lyase synthesis in $N$. salmonicolor shows many similarities to the control of amidase synthesis in Pseudomonas aeruginosa (Brammar \& Clarke, 1964; Smythe \& Clarke, 1972). These authors concluded that aliphatic-amidase synthesis in this Pseudomonad is regulated by induction by amides, and catabolite repression by succinate and other metabolites. Nocardia salmonicolor grows more rapidly on acetate than on any other compound amongst a wide variety of substrates tested, and glucose is amongst the substrates supporting relatively slow growth (Table I). Since the organism shows this substrate preference, it might be predicted that isocitrate lyase would be regulated in a manner different from that in Enterobacteria and that catabolite repression would not involve glucose or glycolytic intermediates. It is possible that isocitrate dehydrogenase activity may also be regulated in a different fashion in $N$. salmonicolor (Fig. 4 ) so that the relative activities of the lyase and the dehydrogenase for isocitrate are appropriate for the substrate(s) presented, in relation to the organism's substrate preference.

It might be expected that the biosynthesis of key enzymes of intermediary metabolism, particularly those involved in anaplerotic pathways, would be regulated in different ways in bacteria from different natural habitats, e.g. Enterobacteria (from the intestine) and Nocardia (from soil). Such differences in regulation would reflect availability of natural substrates, their points of entry into central pathways and the substrate preferences of particular species.

\section{Regulation of isocitrate lyase activity}

A variety of common intermediary metabolites was tested for inhibitory effects on $N$. salmonicolor isocitrate lyase in crude, cell-free extracts. Only PEP had an effect at concentrations that might be physiologically significant, being a typical non-competitive inhibitor with an apparent $K_{i}$ of $\mathrm{I} \cdot 7 \mathrm{mM}$. Whether this effect is in fact significant is doubtful, since addition of pyruvate to cultures growing on acetate did not affect the growth rate, although the organism contains PEP synthase. In contrast, addition of pyruvate at $5 \mathrm{~mm}$ to an Arthrobacter sp. 
growing on acetate inhibited growth completely, and this effect was attributed to inhibition of isocitrate lyase (Wolfson \& Krulwich, 1972). It is possible, however, that pyruvate does not enter $N$. salmonicolor under these circumstances. It is noteworthy that the $K_{i}$ for PEP of the Nocardia enzyme is about 13 -fold greater than that of the E. coli enzyme (Ashworth \& Kornberg, 1963).

Further studies are needed to clarify the mechanism (if any) of fine control of isocitrate lyase in N. salmonicolor.

This work was supported by grants nos. B/RG/06656 and B/RG/6948 from the Science Research Council to I.J.H.

\section{REFERENCES}

ASHWORTH, J. M. \& KornBerg, H. L. (1963). Fine control of the glyoxylate cycle by allosteric inhibition of isocitrate lyase. Biochimica et biophysica acta 73, 519-522.

BaUChOP, T. \& ElSDEN, S. R. (1960). The growth of micro-organisms in relation to their energy supply. Journal of General Microbiology 23, 457-470.

BEEVER, R. E. (1975). Regulation of 2-phosphoenolpyruvate carboxykinase and isocitrate lyase in Neurospora crassa. Journal of General Microbiology 86, 197-200.

BeLL, E. J. \& HeRMAN, N. J. (I967). Effect of succinate on isocitrate lyase synthesis in Mima polymorpha. Journal of Bacteriology 93, 2020-2021.

BenNetT, P. M. \& Holms, W. H. (1975). Reversible inactivation of the isocitrate dehydrogenase of Escherichia coli ML 308 during growth on acetate. Journal of General Microbiology 87, 37-5I.

Brammar, W. J. \& Clarke, P. H. (1964). Induction and repression of Pseudomonas aeruginosa amidase. Journal of General Microbiology 37, 307-319.

COOPER, R. A. \& KorNBERG, H. L. (I967). The direct synthesis of phosphoenolpyruvate from pyruvate by Escherichia coli. Proceedings of the Royal Society B, 168, 263-280.

DAVIS, J. B. \& RAYMOND, R. L. (I96I). Oxidation of alkyl-substituted cyclic hydrocarbons by a Nocardia during growth on $n$-alkanes. Applied Microbiology 9, 383-388.

Dixon, G. H. \& Kornberg, H. L. (1959). Assay methods for key enzymes of the glyoxylate cycle. Biochemical Journal 72, 3P.

Flavell, R. B. \& Woodward, D. O. (197I). Metabolic role, regulation of synthesis, cellular localization, and genetic control of the glyoxylate cycle enzymes in Neurospora crassa. Journal of Bacteriology 105, $200-210$.

Herman, N. J. \& Bell, E. J. (1970). Metabolic control in Acinetobacter sp. I. Effect of $\mathrm{C}_{4}$ versus $\mathrm{C}_{2}$ and $\mathrm{C}_{3}$ substrates on isocitrate lyase synthesis. Canadian Journal of Microbiology 16, 769-774.

HigGins, I. J. \& SaRIaslani, F. S. (1973). Control of isocitrate lyase in Nocardia salmonicolor (N.C.I.B. 970I). Proceedings of the Society for General Microbiology I, 2. I.

HiLdebrandr, W. \& WeIDE, H. (1974). Isocitratlyase von Candida guilliermondii, Stamm HI 7. II. Regelung durch ausgewählte Intermediate des Tricarbonsäurezyklus und Hexoseabbaus. Zeitschrift fïr allgemeine Mikrobiologie I4, 39-46.

HoRECKER, B. L. \& KorNBerg, A. (1948). The extinction coefficients of the reduced band of pyridine nucleotides. Journal of Biological Chemistry 175, 385-390.

HULTMANN, E. (1959). Rapid specific methods for determination of aldosaccharides in body fluids. Nature, London 183, I08-109.

KoRNBERG, H. L. (I966). The role and control of the glyoxylate cycle in Escherichia coli. Biochemical Journal 99, I-II.

Kornberg, H. L., Dennis, J. \& Wilson, E. M. (1964). Regulation of isocitrate lyase synthesis in Achromobacter d-1 5. Biochemical Journal 92, 55 P.

Lowry, O. H., Rosebrough, N. J., Farr, A. L. \& Randall, R. J. (I95I). Protein measurement with the Folin phenol reagent. Journal of Biological Chemistry 193, 265-275.

ROSENBERGER, R. F. (I962). An induction-like effect of acetate on the synthesis of isocitrate lyase. Biochimica et biophysica acta 64, I68-170.

SariasLaNI, F. S., HarPer, D. B. \& Higgins, I. J. (1974). Microbial degradation of hydrocarbons. Catabolism of I-phenylalkanes by Nocardia salmonicolor. Biochemical Journal 140, 3I-45.

SMYthe, P. F. \& ClARKe, P. H. (1972). Catabolite repression of Pseudomonas aeruginosa amidase. Journal of General Microbiology 73, ix.

SUTTON, W. B. (1954). Isolation and properties of a lactic oxidative decarboxylase from Mycobacterium phlei. Journal of Biological Chemistry 210, 309-320.

TRUST, T. J. \& MILLIS, N. F. (1970). The isolation and characterization of alkane-oxidizing organisms and the effect of growth substrate on isocitrate lyase. Journal of General Microbiology 6r, 245-254.

WOLFSON, P. J. \& KRULWICH, T. A. (1972). Inhibition of isocitrate lyase: the basis for inhibition of growth of two Arthrobacter species by pyruvate. Journal of Bacteriology II2, 356-364. 